\title{
Atomic-scale characterization of thermoelectric oxides using high spatial and energy resolution STEM-EELS
}

Quentin M. Ramasse ${ }^{1}$, Demie M. Kepaptsoglou ${ }^{1}$, Jakub D. Baran ${ }^{2}$, Marco Molinari ${ }^{2,3}$, Stephen C. Parker $^{2}$, Teruyasu Mizoguchi ${ }^{4}$, Feridoon Azough ${ }^{4}$ and Robert Freer ${ }^{5}$

1. SuperSTEM Laboratory, Daresbury United Kingdom

2. Department of Chemistry, University of Bath, Claverton Down, Bath, United Kingdom

${ }^{3}$ Institute of Industrial Science, University of Tokyo, Tokyo 153-8505 Tokyo, Japan

4. Department of Chemistry, University of Huddersfield, Huddersfield, HD1 3DH, United Kingdom

5. School of Materials, University of Manchester, Manchester, United Kingdom

Thermoelectric (TE) devices convert heat into electricity and represent an important route for green technologies. They have the potential for making an impact in many fields, such as portable devices (medical applications) and smart grid systems (coupled with batteries and photovoltaics). Metal oxides possess several advantages over traditional thermoelectric (TE) materials including low price, nontoxicity, chemical and thermal stability at high temperature. Furthermore, the chemical richness of their structures ensures that their properties are highly tuneable [1]. Accurate information on the crystal structure and the resulting electronic properties is of paramount importance for understanding and predicting TE oxides' properties reliably as macroscopic quantities like Seebeck coefficient and electronic conductivity are directly related to the electronic states in the vicinity of the Fermi level. Here we use atomic-scale, high energy resolution scanning transmission electron microscopy (STEM) and electron energy loss spectroscopy to inform theoretical predictions from density functional theory electronic band-structure calculations combined with the Boltzmann transport theory, to a number of highly promising TE oxide systems.

In particular, we investigate the misfit-layered layered cobalt oxide bismuth strontium cobaltite $\left[\mathrm{Bi}_{0.87} \mathrm{SrO}_{2}\right]_{2}\left[\mathrm{CoO}_{2}\right]_{1.82}$ (BSCO). We shed further light on the structure of this material, whose high efficiency makes it one of the most exciting TE oxides, highlighting the common presence of stacking faults, finding that changes in the relative arrangements of the $\mathrm{CoO}_{2}$ and $\mathrm{BiSrO}$ layers can easily arise as their formation requires a very low energetic cost. We further demonstrate that Bi deficiency has a paramount importance on BSCO's electronic, magnetic and transport properties. Atomic-layer-resolved electron energy loss spectroscopy shows how $\mathrm{Bi}$ vacancies lead to the hole-doping of the $\mathrm{CoO}_{2}$ layer (figure 1), which our theoretical modelling then demonstrates is in turn responsible for the high positive Seebeck coefficient of the material measured experimentally. [2]

Furthermore, we reveal the effect of the A-site occupancy in the structure and electronic structure on an A-site deficient perovskite system based on the $\mathrm{Nd}_{2 / 3} \mathrm{TiO}_{3}$ double perovskite. This system, a candidate for thermoelectric applications [3], has attracted significant attention due to the presence of a peculiar superstructure originating in part due to cation vacancy ordering of the A-site [4]. Using atomically resolved monochromated core loss EELS measurements, acquired with an energy resolution better than $90 \mathrm{meV}$ with a Nion UltraSTEM 100MC, it is possible to map individual components of the $\mathrm{Ti} \mathrm{L}_{2,3}$ and $\mathrm{O} \mathrm{K}$ near edge fine structures (ELNES). First-principles multiplet calculations are used to explain subtle changes in the ELNES, and associate them predominantly with Coulombic interactions from the A-sites. Annular Bright Field Imaging can then correlate the presence of tilting domains in the $\mathrm{TiO}_{6}$ sub lattice with these electronic structure changes observed by EELS. 


\section{References:}

[1] R. Funahashi, T. Barbier, E. Combe, Journal of Materials Research 30 (2015), p. 2544.

[2] J.D. Baran et al., Chem. Mat. 28 (2016) p.7470.

[3] S. Jackson et al., J. Electron. Mater. 43 (2014) p.2331.

[4] F. Azough et al., Chem. Mater 27 (2015) p.497.

[5] SuperSTEM is the U.K's national facility for Aberration Corrected STEM funded by EPSRC.
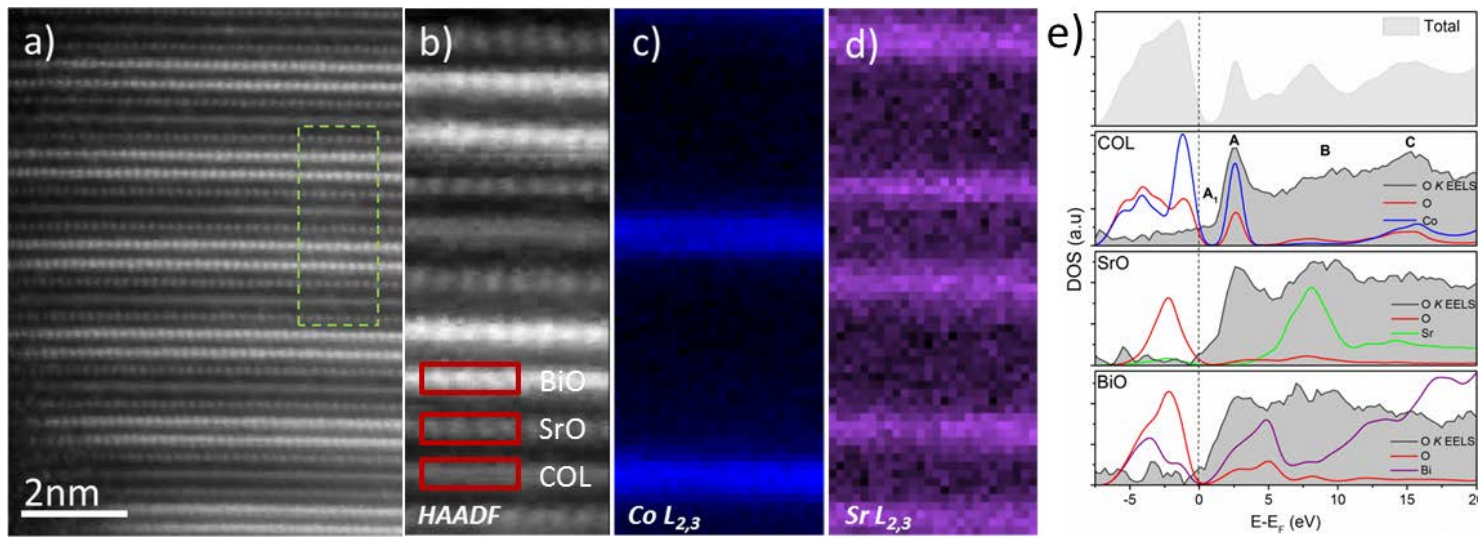

Figure 1. a) HAADF image of the bismuth-deficient misfit-layered layered $\left[\mathrm{Bi}_{0.87} \mathrm{SrO}_{2}\right]_{2}\left[\mathrm{CoO}_{2}\right]_{1.82}$ (BSCO) acquired along the [110] zone axis. The area marked by dashed lines was used to record EELS maps of the structure (c-d). e) Total calculated DOS and site-projected DOS on the Co and O of COL, Sr and $\mathrm{O}$ of the $\mathrm{SrO}$ layer and projected on $\mathrm{Bi}$ and $\mathrm{O}$ of $\mathrm{BiO}$ layer, as labeled on $\mathrm{b}$ ). The Fermi level is set to $0 \mathrm{eV}$. The p-DOS is plotted against the experimental $\mathrm{O} \mathrm{K}$ edges for the corresponding layers [2].

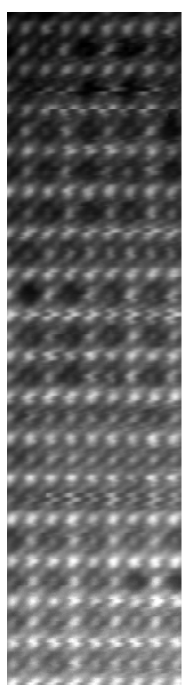

HAADF

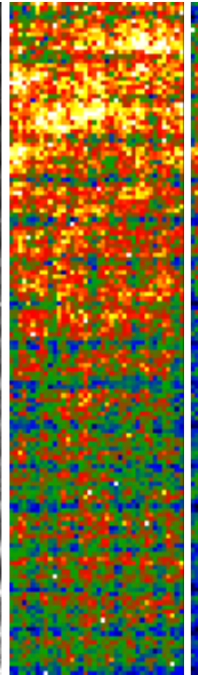

a'

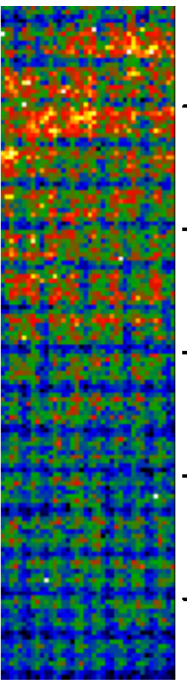

a"

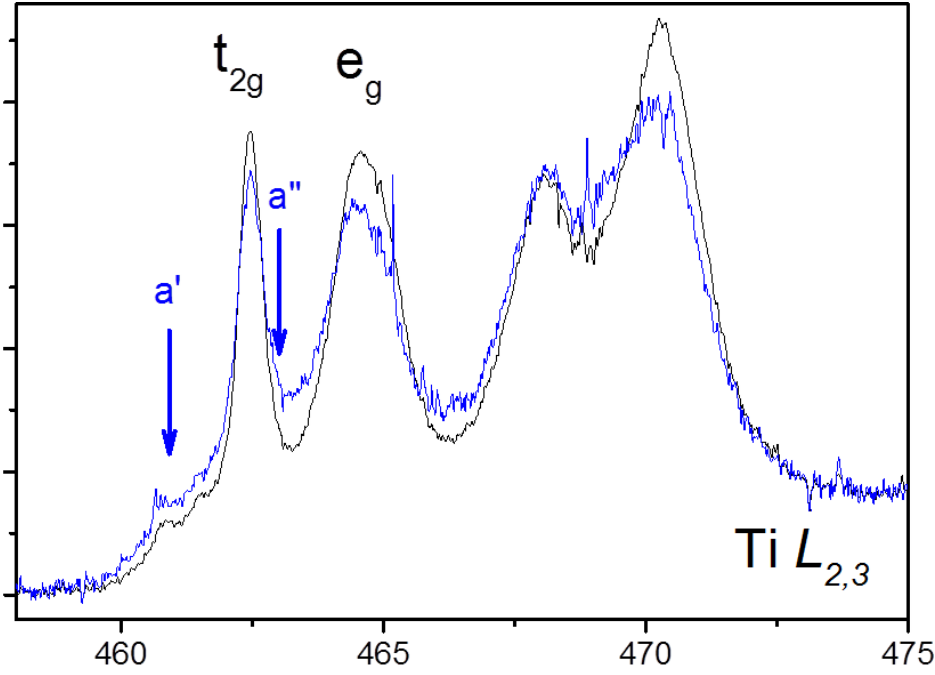

energy $(\mathrm{eV})$

Figure 2. Monochromated core loss EELS maps of individual components a' and a" of the Ti $\mathrm{L}_{2,3}$ ELNES and relative intensity changes related to local electronic charge variations in the vacancy-rich area seen at the top of the HAADF image (left) recorded simultaneously with the EELS 